\title{
Melanoma ungueal e vitiligo: relato de caso
}

\section{Ungueal melanoma and vitiligo: case report}

\author{
Ciléia I.C. Oliveira ${ }^{1}$, Cid C.S. Alcântara², Fernanda N. M. Leal ${ }^{3}$, Willy Okoba ${ }^{4}$
}

\begin{abstract}
RESUMO
O melanoma ungueal é uma forma de apresentação rara do melanoma. É frequente o diagnóstico tardio. Geralmente afeta polegar ou hálux e a maioria dos pacientes é de meia idade ou idosos de pele escura. A associação entre melanoma e vitiligo é tem sido descrita há mais de 50 anos e os pacientes acometidos tem apresentado maior sobrevida sugerindo resposta imune ao melanoma. O objetivo deste relato é descrever um caso de melanoma ungueal com surgimento de linfonodo inguinal e vitiligo associado diagnosticado tardiamente com posterior evolução para doença sistêmica.
\end{abstract}

Palavras-chave: Melanoma. Vitiligo. Doenças da Unha. Recidiva.

\begin{abstract}
Subungual melanoma is a rare clinical entity of melanoma. It usually involves an unsuspecting lesion in the thumb or big toe, thus late diagnosis. Majority of patients are middle-aged or older, usually of dark skin ethnicity. The association between melanoma and vitiligo has been described for over 50 years, showing increased survival rates in affected patients suggesting immune response to melanoma. The objective of this report is to describe a clinical case of nail melanoma, presenting as enlargened unilateral inguinal lymph node associated with rapid and progressive vitiligo. However, investigations proved late diagnosis, with subsequent progression of the disease.
\end{abstract}

Keywords: Melanoma. Vitiligo. Nail Diseases. Recurrence.

\section{Introduçåo}

O melanoma é um câncer de pele potencialmente fatal que se origina dos melanócitos e pode se disseminar através dos linfonodos e circulação.
O melanoma lentiginoso acral é raro em caucasianos, mas é o mais comum em pacientes orientais e negros. Ao contrário de outros subtipos da neoplasia ele parece não estar associado à radiação ultravioleta ou a nevos. A localização preferencial é sob a
1. Estudante de Medicina da Universidade Federal do Ceará

2. Mestrado em Saúde Pública e Residência em Clínica Médica pela Universidade Federal do Ceará

3. Residência em Dermatologia pela Université Claude Bernarde Lyon. Professora Substituta da Universidade Federal do Ceará 4. Residente de Cirurgia da Universidade Federal do Ceará

Conflito de interesses:

Os autores declaram não haver conflito de interesses
Correspondencia

Cid Carlos Soares de Alcântara Universidade Federal do Ceará, Faculdade de Medicina Rua Alexandre Baraúna, 949 - Rodolfo Teófilo CEP: 60430-160 - Fortaleza, CE

Artigo recebido em 05/05/2014 Aprovado para publicação em 03/02/2015 
região palmo-plantar e unhas sendo comumente o diagnóstico tardio. ${ }^{1}$

O melanoma do aparelho ungueal é uma variante do melanoma lentiginoso acral. A incidência varia entre $0,7 \%$ e $3,5 \%$ entre os casos de melanoma. Costuma afetar mais comumente polegar ou hálux. $\mathrm{O}$ trauma pode ser um fator precipitante. A maioria dos pacientes é de meia idade ou idosos de pele escura. Dor e desconforto são sintomas pouco frequientes ${ }^{2}$. Pode determinar deformidade da lâmina ungueal quando tiver origem no leito ungueal, porém, grande parte surge casualmente como uma lesão pigmentada no polegar ou no hálux. ${ }^{3}$. Muitas vezes é diagnosticado como nevo juncional, hematoma subungueal ou mesmo onicomicose. ${ }^{4}$

O vitiligo é uma doença de despigmentação adquirida, caracterizada por perda de melanócitos da epiderme. Acomete cerca de $1 \%$ da população, comprometendo de modo semelhante homens e mulheres, preferencialmente entre 10 e 30 anos de idade. $\mathrm{O}$ curso da doença é geralmente progressivo com fases de estabilização. ${ }^{5}$ Uma das hipóteses mais populares e aceita considera o vitiligo como uma doença autoimune tendo como alvo as células pigmentares. ${ }^{6}$

A associação entre melanoma e vitiligo tem sido descrita há mais de 50 anos, apesar das manifestações clínicas opostas. Nesses indivíduos frequentemente são encontrados metástases para linfonodos regionais, sendo capazes de sobreviver com o tumor por período mais longo do que os pacientes portadores de melanoma sem vitiligo sugerindo melhor prognóstico. ${ }^{7,8}$

O objetivo deste estudo foi relatar um caso de melanoma ungueal diagnosticado tardiamente após surgimento vitiligo e metástases.

\section{Relato de Caso}

Paciente do sexo masculino, 56 anos, negro, agricultor, com história de que há nove anos apresentou uma lesão ungueal escurecida no hálux esquerdo, que evoluiu com ulcerações periungueais que infectaram. Procurou atendimento médico onde foi prescrito antibioticoterapia, sem sucesso, sendo necessária a amputação do hálux esquerdo (figura 1) para controle do foco infeccioso, no entanto, não foi realizado biópsia da peça. Dessa forma não foi identificada a doença que originou o foco infeccioso. Alguns meses após a amputação do hálux houve o surgimen- to de linfonodomegalia inguinal à esquerda com aumento progressivo (figura 2), no entanto, o paciente recebeu o diagnóstico de hérnia inguinal sem investigação adicional. Há três anos surgiram manchas hipocrômicas em face e membros inferiores (figuras 2 e 3), de caráter progressivo. Procurou assistência médica recebendo o diagnóstico de vitiligo, sendo tratado com Metoxisaleno durante um ano, sem sucesso. Há cinco meses passou a apresentar tosse produtiva com secreção escurecida associado a dispnéia aos grandes esforços que progrediu para dispneia aos moderados esforços. Houve perda ponderal de oito quilos nesse período (12,5\% de perda). Procurou assistência médica no serviço de pneumologia da Santa Casa com dispnéia e presença de linfonodo inguinal esquerdo aumentado de volume com cinco centímetros de diâmetro e consistência endurecida. Foi internado para esclarecimento diagnóstico. Os resultados dos exames de maior relevância para a investigação diagnóstica encontram-se na Tabela 1.

Após resultados dos exames o paciente foi amplamente examinado em busca da lesão inicial de melanoma que evoluiu para metástase. Através da anamnese e exame físico minuciosos foi possível compreender que se tratava de um melanoma ungueal não diagnosticado, que evoluiu com disseminação para linfonodos e surgimento de vitiligo paraneoplásico. $\mathrm{O}$ paciente foi encaminhado ao setor de oncologia, sendo iniciado tratamento quimioterápico.

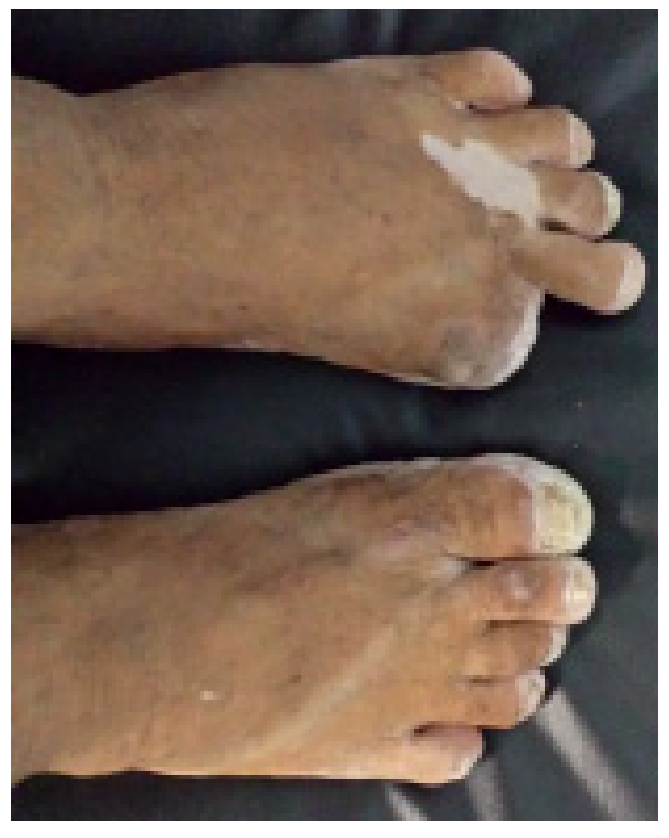

Figura 1. Vitiligo em pés e hálux esquerdo amputado. 


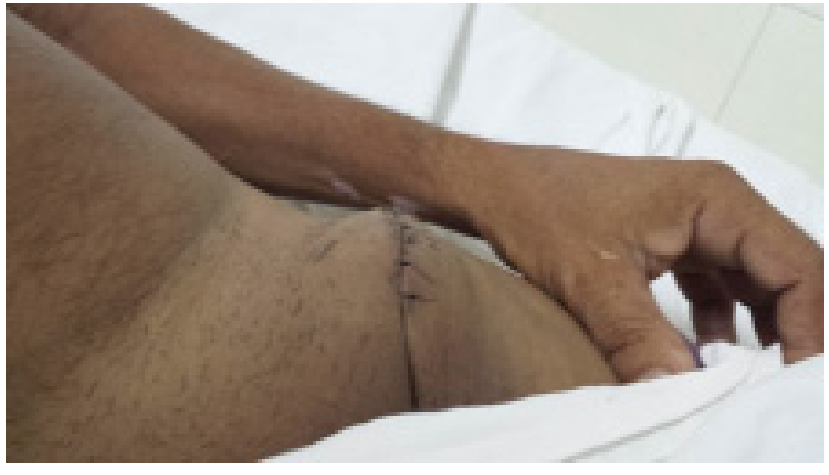

Figura 2. Linfonodo inguinal esquerdo biopsiado.

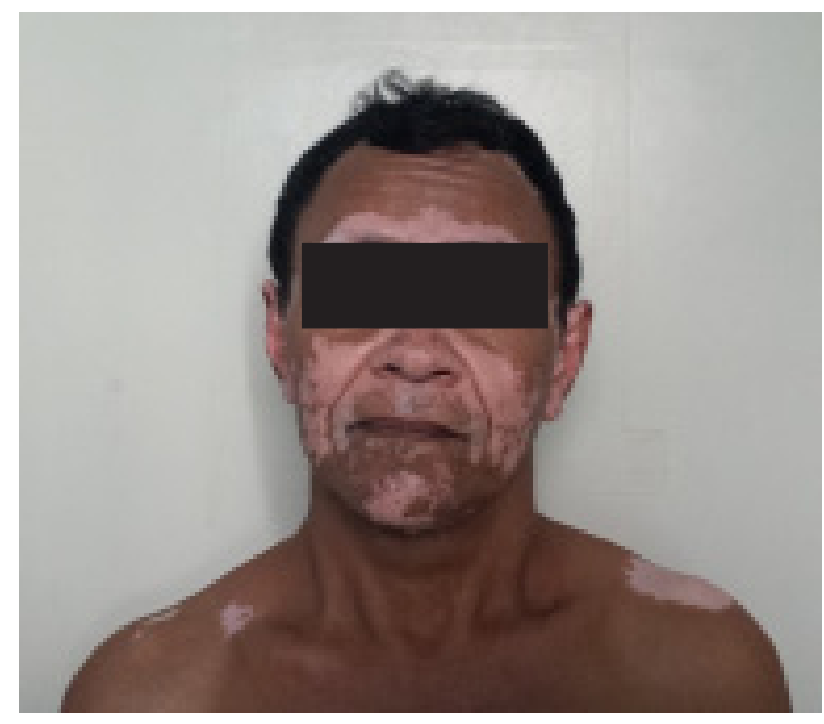

Figura 3. Vitiligo em face e ombros.

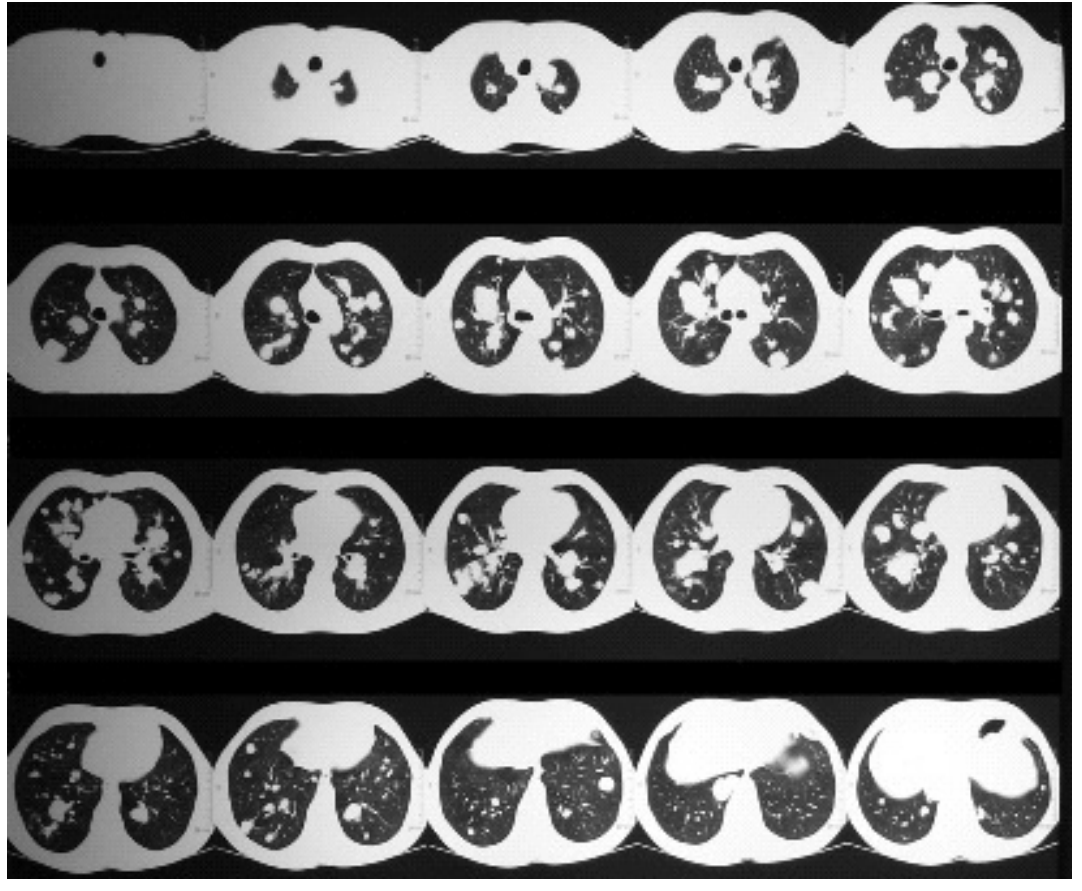

Figura 4. Tomografia de tórax evidenciando múltiplas metástases pulmonares. 


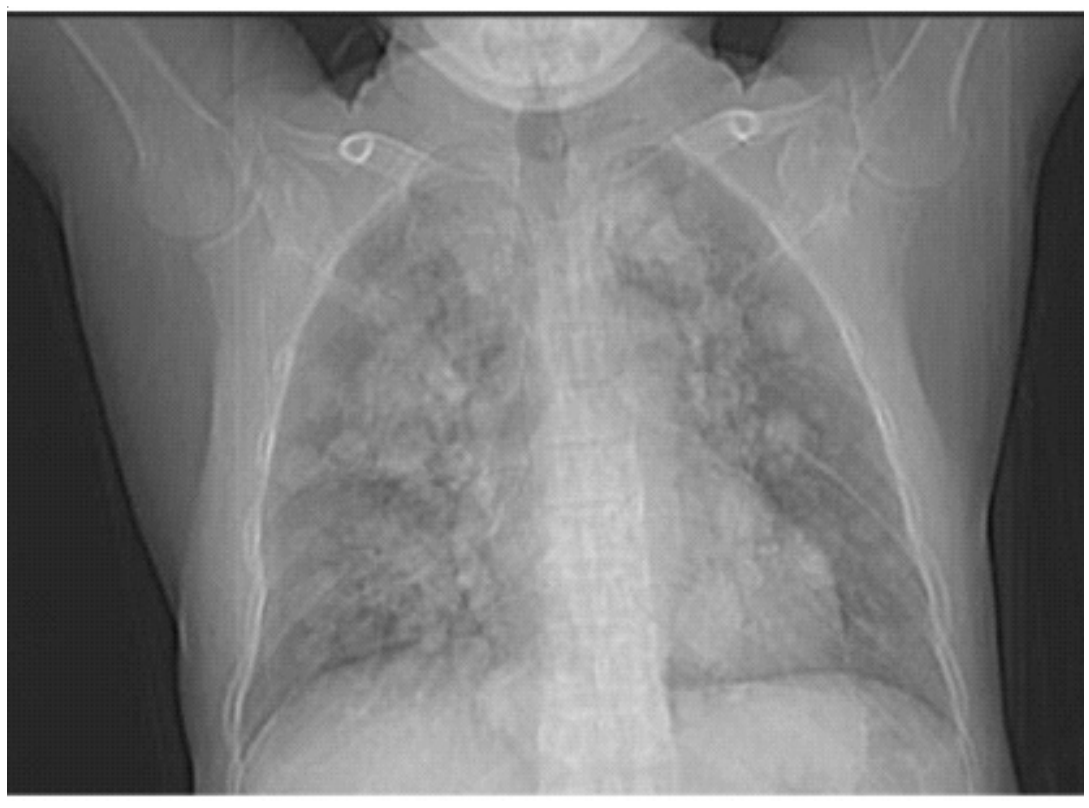

Figura 5. Radiografia de tórax mostrando múltiplos nódulos pulmonares bilaterais

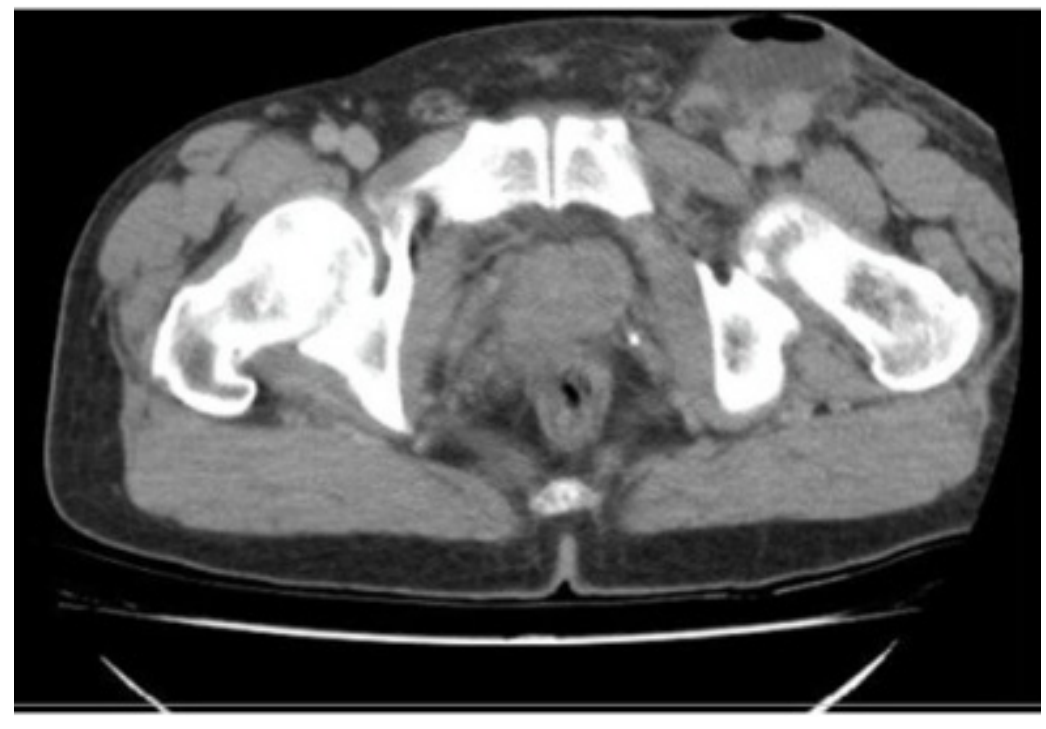

Figura 6. Tomografia de pelve com contraste visualiza imagem compatível com linfonodo inguinal à esquerda aumentado

Tabela 1: Exames realizados durante o internamento

\begin{tabular}{ll}
\hline Exames & Resultados \\
\hline Radiografia de Tórax & Imagens nodulares difusas e extensas (Figura 5) \\
Tomografia de Tórax & Nódulos compatíveis com metástase pulmonar (Figura 4) \\
Tomografia de Pelve & Linfonodomegalia inguinal esquerda medindo $4 \mathrm{~cm} \mathrm{X} 4 \mathrm{~cm} \times 3,2 \mathrm{~cm}($ Figura 6) \\
$\begin{array}{l}\text { Biópsia excisional de linfonodo } \\
\text { inguinal esquerdo }\end{array}$ & Melanoma metastático extracapsular \\
\hline
\end{tabular}




\section{Discussão}

O relato do caso mostra a importância do exame físico cuidadoso e a investigação de lesões de difícil cicatrização e de linfonodos identificados. $\mathrm{Na}$ evolução da história clínica do paciente esses achados foram negligenciados sem investigação diagnóstica adicional para exclusão de causas neoplásicas. $\mathrm{O}$ paciente apresentou longa evolução com duração de nove anos entre o surgimento das primeiras manifestações de doença e a expressão de sintomas sistêmicos como tosse, dispnéia e perda de peso. É importante ressaltar que o diagnóstico diferencial do melanoma ungueal é feito com hemorragia subungueal, pigmentação étnica, lentigo ungueal ou pigmentação medicamentosa sendo fundamental para o diagnóstico precoce. Este é realizado através da avaliação clínica, dermatoscópica e biópsia da lesão. As manchas hipercrômicas possuem um padrão irregular das linhas longitudinais e pode haver o sinal de Hutchinson (pigmentação da cutícula na zona que corresponde à melanoníquia longitudinal). ${ }^{9}$

Ao contrário do melanoma cutâneo em que $80 \%$ dos casos são TNM I no diagnóstico, apenas $20 \%$ dos casos de melanoma ungueal estão no estágio I do TNM (classificação para tumor primário, linfonodos regionais e metástases) no momento do diagnóstico. ${ }^{10}$

Para pacientes com melanoma a chance de desenvolver vitiligo é 7-10x maior comparado com a população geral especialmente quando submetidos a terapia imunológica. ${ }^{11}$

As diferenças clínicas entre vitiligo associado a melanoma e o vulgar não são bem definidas: alguns autores consideram idênticos ${ }^{12}$ enquanto outros verificaram que o vitiligo associado ao melanoma tem espectro de manifestações clínicas mais variadas. ${ }^{13}$

A patogênese da despigmentação no melanoma é provavelmente autoimune com anticorpos contra antígenos dos melanócitos, como a tirosinase encontrados no soro de uma proporção significativa destes patientes. ${ }^{14,15}$ Em estudos in vitro, soros de pacientes com vitiligo são capazes destruir melanócitos huma$\operatorname{nos}^{16}$. O mecanismo de inibição da imunidade é explicado pelo antígeno quatro associado com linfócito T citotóxico (CTLA-4). Este normalmente é encontrado em estoques intracelulares dentro de células $T$ e á transportado para a superfície da célula após ativação da célula $T$ através do receptor de células $T$ (RCT). Uma vez expressas na superfície das células, o CTLA-4 compete com a molécula CD28, CD80 e CD86 nas células apresentadoras de antígenos para fornecer um sinal negativo para o RCT. O ligante para CTLA-4 não é expresso em células tumorais. ${ }^{17}$

$\mathrm{O}$ vitiligo seria um marcador da imunidade contra as células neoplásicas do melanoma levando a um impacto positivo na sobrevida desses pacientes. ${ }^{18}$

O tratamento depende do estadiamento e tem a cirurgia como melhor opção. O prognóstico costuma ser reservado devido ao comportamento agressivo e diagnóstico tardio do melanoma na maioria dos casos. ${ }^{19}$

\section{Conclusăo}

O exame clínico cuidadoso associado ao exame dermatoscópico e histopatológico das lesões pigmentares cutâneas é de grande importância na identificação de lesões cutâneas e detecção do melanoma lentiginoso acral, pois o tratamento instituído precocemente pode melhorar o prognóstico desses pacientes. A presença do vitiligo pode ser manifestação de doença subjacente e sua presença pode indicar diagnóstico tardio de neoplasia associada.

\section{Referências}

1. Du Vivier A. Atlas de dermatologia. 3 ed. Rio de Janeiro: Elsevier; 2004 p.215

2. Langley RG, Barnhill RL, Fitzpatrick TB. Fitzpatrick's Dermatology in general medicine. 5nd ed vol I. New York: McGrawHill; 1999. p. 1080-116.

3. O'Toole E, Stephens R, Young MM, Tanner A, Barnes L. Subungual Melanoma: a relation to direct injury? J Am Acad Dermatol. 1995; 33:525-8.

4. Glat PM, Spector JA, Roses DF, Shapiro RA, Harris MN, Beasley RW et al. The management of pigmented lesions of the nail bed. Ann Plast Sur. 1996; 37:25-34.

5. Ongenae K, Van Geel N, Naeyaert JM. Evidence for an autoimmune pathogenesis of vitiligo. Pigment Cell Res. 2003;16:90-100.

6. Ortonne JP, Bose SK. Vitiligo: where do we stand? Pigment Cell Res. 1993; 6:61-72.

7. Nordlund JJ, Kirkwood JM, Forget BM, Milton G, Albert DM, Lerner AB. Vitiligo in patients with metastatic melanoma: $A$ good prognostic sign. J Am Acad Dermatol. 1983; 9:689-96.

8. Byrne KT, Turk MJ. New perspectives on the role of vitiligo in immune responses to melanoma. Oncotarget. 2011;2:68494.

9. R. P. Braun, L. Thomas. Atlas de dermatoscopia. 1 ed. Rio de Janeiro: Revinter; 2007 p. 140-41

10. Levit EK, Kagen MH, Scher RK, Grossman M, Altman E. The $A B C$ rule for clinical detection of subungual melanoma. J Am Acad Dermatol. 2000; 42(2 Pt 1):269-74. 
11. Jacobs JF, Aarntzen EH, Sibelt LA, Blokx WA, Boullart AC, Gerritsen MJ, et al. Vaccine-specific local T cell reactivity in immunotherapy associated vitiligo in melanoma patients. Cancer Immunol Immunother. 2009;58:145-51. doi: 10.1007/ s00262-008-0506-5. Epub 2008 Apr 5.

12. Hartmann A, Bedenk C, Keikavoussi P, Becker JC, Hamm H, Bröcker EB. Vitiligo and melanoma-associated hypopigmentation (MAH): shared and discriminative features. J Dtsch Dermatol Ges. 2008;6:1053-9. doi: 10.1111/j.16100387.2008.06755.x. Epub 2008 May 7.

13. Koh HK, Sober AJ, Nakagawa H, Albert DM, Minm MC, Fitzpatrick TB. Malignant melanoma and vitiligo-like leukoderma: an electron microscopic study. J Am Acad Dermatol. 1983;9:696-708.

14. Kemp EH, Waterman EA, Hawes BE, O'Neill K, Gottumukkala RV, Gawkrodger DJ, Weetman AP, Watson PF. The melaninconcentrating hormone receptor 1 , a novel target of autoantibody responses in vitiligo. J Clin Invest. 2002;109:92330.
15. Song YH, Connor E, Li Y, Zorovich B, Balducci P, Maclaren N. The role of tyrosinase in autoimmune vitiligo. Lancet. 1994;15:344(8929):1049-52.

16. Gilhar A1, Zelickson B, Ulman Y, Etzioni A. In vivo destruction of melanocytes by the $\mathrm{IgG}$ fraction of serum from patients with vitiligo. J Invest Dermatol. 1995;105:683-6.

17. Gyorki DE, Callahan M, Wolchok JD, Ariyan CE. The delicate balance of melanoma immunotherapy. Clinical \& Translational Immunology. (2013) 2, e5; doi:10.1038/cti.2013.5 Published online 9 August 2013

18. Daneshpazhooh M, Shokoohi A, Dadban A, Raafat J. The course of melanoma-associated vitiligo: report of a case. Melanoma Res. 2006;16:371-3.

19. Paul E, Kleiner H, Bodeker RH. Epidemiology and prognosis of subungual melanoma. Hautarzt. 1992; 43:286-90. 